\title{
On the Nonlocality of the Quantum Channel in the Standard Teleportation Protocol
}

\author{
Rob Clifton \\ Departments of Philosophy and History and Philosophy of Science, \\ 10th floor, Cathedral of Learning, University of Pittsburgh, \\ Pittsburgh, PA 15260, USA. \\ email: rclifton+@pitt.edu \\ Damian Pope \\ Department of Physics, University of Queensland \\ Brisbane 4072, Queensland, Australia \\ email: pope@physics.uq.edu.au
}

(October 29, 2018)

\begin{abstract}
By exhibiting a violation of a novel form of the Bell-CHSH inequality, Żukowski has recently established that the quantum correlations exploited in the standard perfect teleportation protocol cannot be recovered by any local hidden variables model. Allowing the quantum channel state in the protocol to be given by any density operator of two spin-1/2 particles, we show that a violation of a generalized form of Żukowski's teleportation inequality can only occur if the channel state, considered by itself, violates a Bell-CHSH inequality. On the other hand, although it is sufficient for a teleportation process to have a nonclassical fidelity - defined as a fidelity exceeding $2 / 3$ - that the channel state employed violate a Bell-CHSH inequality, we show that such a violation does not imply a violation of Żukowski's teleportation inequality or any of its generalizations. The implication does hold, however, if the fidelity of the teleportation exceeds $2 / 3(1+1 / 2 \sqrt{2}) \approx .90$, suggesting the existence of a regime of nonclassical values of the fidelity, less than .90, for which the standard teleportation protocol can be modelled by local hidden variables.
\end{abstract}

PACS numbers: 03.65.Bz, 03.67.-a

\section{INTRODUCTION}

It is well-known that the quantum correlations predicted by pure entangled states violate Bell-CHSH inequalities [1] and, therefore, cannot be recovered by any local hidden variables model. Yet these same correlations cannot by themselves be used to transmit information between the locations of the entangled systems. This has sometimes been taken to suggest that quantum correlations are not inherently 'nonlocal', but simply 'nonclassical'. On the other hand, it is a striking fact that quantum correlations can used, as in quantum teleportation, to increase the information carrying capacity of a classical channel. This new operational manifestation of quantum entanglement invites a deeper analysis of the extent to which the quantum teleportation process itself can be modelled classically, and what role, if any, nonlocality must play in explaining the success of teleportation.

The standard quantum teleportation protocol runs as follows [2]. Consider three qubit systems 1,2 , and 3, fix an arbitrary direction in space to define the $z$-direction for each qubit, and let $|\uparrow\rangle$ and $|\downarrow\rangle$ denote the eigenstates of $\sigma_{z}$. We can imagine that all three qubits are initially in the possession of someone named Clare, who follows the instructions of Alice and Bob to prepare qubits $2+3$ in a quantum channel state given by some density operator $D$ of their choice, and prepares qubit 1 in a pure state $|\phi\rangle$, unknown to Alice or Bob. Clare then feeds qubits
2 and 3 to Alice and Bob, respectively, to use as their "quantum channel", and Clare passes qubit 1 to Alice so that she can teleport its unknown state to Bob. To execute the protocol, Alice measures the "Bell operator" on qubits $1+2$, which has eigenstates [3]:

$$
\begin{aligned}
& \left|\Phi_{ \pm}\right\rangle=1 / \sqrt{2}(|\uparrow\rangle|\uparrow\rangle \pm|\downarrow\rangle|\downarrow\rangle), \\
& \left|\Psi_{ \pm}\right\rangle=1 / \sqrt{2}(|\uparrow\rangle|\downarrow\rangle \pm|\downarrow\rangle|\uparrow\rangle),
\end{aligned}
$$

and she obtains one of four results $n=1, \ldots, 4$ corresponding to the eigenstates above, with probabilities $p_{n}$. For each outcome $n$, Alice then sends classical information, via (say) a normal telephone call, instructing Bob to perform a corresponding unitary transformation $U_{n}$ on his qubit 3. Having followed Alice's instructions, Bob's qubit will be left in one of four states $D_{n}$.

If the quantum channel state $D$ is a maximally entangled pure state, the unitaries $U_{n}$ can always be chosen so that no matter what Alice's Bell operator measurement outcome, Bob will have prepared his particle by the completion of the protocol in exactly the same state $D_{n}=|\phi\rangle\langle\phi|(n=1, \ldots, 4)$ as the state of qubit 1 that Alice teleported. For example, when $D$ is the singlet state, qubit 3 has an equal probability of being in one of the four states $|\phi\rangle\left\langle\phi\left|, \sigma_{x}\right| \phi\right\rangle\left\langle\phi\left|\sigma_{x}, \sigma_{y}\right| \phi\right\rangle\left\langle\phi\left|\sigma_{y}, \sigma_{z}\right| \phi\right\rangle\langle\phi| \sigma_{z}$, so that Bob's performing, respectively, one of the four unitary transformations

$$
U_{1}=I \quad\left(\left|\Psi_{-}\right\rangle\right), U_{2}=\sigma_{x}\left(\left|\Phi_{-}\right\rangle\right),
$$




$$
U_{3}=\sigma_{y}\left(\left|\Phi_{+}\right\rangle\right), \quad U_{4}=\sigma_{z}\left(\left|\Psi_{+}\right\rangle\right),
$$

suffices for perfect teleportation.

For a mixed and/or non-maximally entangled quantum channel state $D$, perfect teleportation of an unknown state cannot be achieved (cf., e.g., 凷). However, one can introduce a natural measure of success for any fixed "strategy" $n \mapsto U_{n}$ of associating unitary operators $U_{n}$ with Alice's Bell operator outcomes. This measure is the fidelity $\mathcal{F}_{\left\{U_{n}\right\}}(D)$ of transmission to Bob, given by the uniform average, over all possible unknown states $|\phi\rangle$, of the quantity $\sum_{n=1}^{4} p_{n} \operatorname{Tr}\left(D_{n}|\phi\rangle\langle\phi|\right)$ [5], [6]. Taking the supremum of $\mathcal{F}_{\left\{U_{n}\right\}}(D)$ over all possible strategies for associating unitary operators $U_{n}$ with Alice's Bell operator outcomes, one obtains a number, $\mathcal{F}_{\max }(D)$, representing the maximum achievable fidelity of teleportation for a given fixed quantum channel state $D$ [7]. A density matrix $D$ is then regarded as useful for nonclassical teleportation just in case $\mathcal{F}_{\max }(D)>2 / 3$, where $2 / 3$ is the maximum achievable fidelity without the quantum channel, or when a classical channel is substituted in its place [5], [8].

Let us return, for the moment, to the case of perfect teleportation, where $D$ is a maximally entangled pure state and $\mathcal{F}_{\text {max }}(D)=1$. The striking thing is that Alice only directly communicates to Bob two classical bits of information when she conveys to him one of the four integers $n=1, \ldots, 4$. (We assume, as usual, that they have agreed in advance on a strategy $n \mapsto U_{n}$.) Indeed, one might think that Bob's sure-fire reconstruction of the unknown state $|\phi\rangle$ as a state of his qubit 3 entails that he must (somehow) have actually received a full qubit's worth of information (i.e., the information contained in specifying the two of infinitely many possible real numbers needed to fix the normalized expansion coefficients of the state $|\phi\rangle)$. A natural, though perhaps naive, explanation would be that the correlations inherent in a maximally entangled pure state carry the extra information to Bob instantaneously and nonlocally when that state "collapses" as a result of Alice's Bell operator measurement. Indeed, the view that the quantum channel itself carries some share of the net information received by Bob appears to have been favoured by the inventors of teleportation ( [2], p. 1896; cf. also [5], p. 797), and is the springboard for a number of different explanations of teleportation from the point of view of particular interpretations of quantum theory ( [9]- [1] ). There are sceptics, however, who have challenged the view that perfect teleportation requires nonlocal information transfer of any sort ( $12|-| 14])$. Moreover, one should bear in mind that the information about the unknown state $|\phi\rangle$ "carried" by Bob's qubit at the completion of a single run of the protocol is not actually accessible to him, since it is well-known that there is no way for him to discern the quantum-mechanical state of a single system.

In the case of explaining imperfect nonclassical tele- portation, where $1>\mathcal{F}_{\max }(D)>2 / 3$, the situation is more complicated, and appears to favour the sceptics. While $D$ must still be entangled (i.e., not a convex combination of product states of $2+3), D$ can still satisfy all Bell-CHSH inequalities [5]

$$
\left|\left\langle\bar{\sigma}_{1} \otimes\left(\sigma_{1}+\sigma_{2}\right)+\bar{\sigma}_{2} \otimes\left(\sigma_{1}-\sigma_{2}\right)\right\rangle_{D}\right| \leq 2,
$$

where the $\bar{\sigma}$ 's and $\sigma$ 's denote arbitrary spin observables of qubits 2 and 3 , respectively, and $\langle\cdot\rangle_{D}$ denotes expectation value in the state $D$. More precisely, if we let $\beta(D) \in[2,2 \sqrt{2}]$ be the maximum over all Bell-CHSH expressions of the above form, then $\mathcal{F}_{\text {max }}(D)>2 / 3$ does not imply $\beta(D)>2$, even though the converse implication does hold [7]. So nonclassical teleportation is possible even in the absence of an independent argument for the nonlocality of the quantum channel state via Bell's theorem. Notwithstanding this, one could adopt the view that the condition $\mathcal{F}_{\max }(D)>2 / 3$ itself should be viewed as sufficient for thinking of the channel state $D$ as nonlocal (cf. [5], p. 799). However, the sceptic is likely to point out that this gives further support to his view that nonclassical teleportation can occur without the involvement of nonlocality (cf. [12], p. 6).

All parties to this discussion are agreed that good teleportation is made possible by the fact that the correlations of an entangled quantum channel state $D$ are nonclassical. They only diverge on the question of whether or not the standard teleportation protocol necessarily involves nonlocality. Recently, Żukowski 115 has made an interesting attempt to force this issue of nonlocality by asking an extremely relevant question: Can the correlations of $D$ that are actually involved in the standard teleportation protocol be recovered in a local hidden variables model? Żukowski attacks this question by making a novel use of a Bell-CHSH inequality, which we explain in the next section. The two sections following, Sections III] and IV, investigate the connection between a generalized form of Żukowski's teleportation inequality and the standard Bell-CHSH inequality for the channel state. In particular, we show that a violation of the former implies that the channel state satisfies $\beta(D)>2$, but that $\beta(D)>2$ does not imply a violation of even our generalized form of Żukowski's teleportation inequality. However, in Section $\mathrm{V}$ we show that such a violation occurs whenever the fidelity $\mathcal{F}_{s t}(D)>2 / 3(1+1 / 2 \sqrt{2}) \approx .90$, where $\mathcal{F}_{s t}$ is the fidelity associated with the standard choice for Bob's unitary operators given in (3). The implications of these results for the interpretation of quantum teleportation as nonlocal are discussed in Section VI.

\section{II. ŻUKOWSKI'S ARGUMENT SIMPLIFIED}

In effect, Żukowski considers the following pair of \pm 1 valued functions of Alice's Bell operator: 


$$
\begin{aligned}
A_{1}= & \left|\Psi_{-}\right\rangle\left\langle\Psi_{-}|+| \Phi_{-}\right\rangle\left\langle\Phi_{-}\right| \\
& -\left|\Psi_{+}\right\rangle\left\langle\Psi_{+}|-| \Phi_{+}\right\rangle\left\langle\Phi_{+}\right|, \\
A_{2}= & \left|\Psi_{+}\right\rangle\left\langle\Psi_{+}|+| \Phi_{-}\right\rangle\left\langle\Phi_{-}\right| \\
& -\left|\Psi_{-}\right\rangle\left\langle\Psi_{-}|-| \Phi_{+}\right\rangle\left\langle\Phi_{+}\right| .
\end{aligned}
$$

The operator $A_{1}\left(A_{2}\right)$ coincides with the observable that represents the first (second) component of the vector observable $\vec{A}$ introduced by Żukowski ( [15], Eqn. (12)). It is easy to see that the information obtained in a joint measurement of $A_{1}$ and $A_{2}$ on qubits $1+2$ is equivalent to the information one would obtain through a single Bell operator measurement. So we can equally well think of Alice as jointly measuring the observables $A_{j}(j=1,2)$ in her attempt to teleport the unknown state to Bob.

Now take $D$ to be the maximally entangled state $\left|\Phi_{+}\right\rangle\left\langle\Phi_{+}\right|$, consider two alternative unknown states given by

$$
\begin{aligned}
& \left|\phi_{1}\right\rangle=\frac{1}{\sqrt{2}}(|\uparrow\rangle+|\downarrow\rangle), \\
& \left|\phi_{2}\right\rangle=\frac{1}{\sqrt{2}}(|\uparrow\rangle+i|\downarrow\rangle),
\end{aligned}
$$

and consider the following two alternative spin components of qubit 3 ,

$$
\begin{aligned}
& \sigma_{1}=e^{-i \pi / 4}|\uparrow\rangle\left\langle\downarrow\left|+e^{i \pi / 4}\right| \downarrow\right\rangle\langle\uparrow|, \\
& \sigma_{2}=e^{i \pi / 4}|\uparrow\rangle\left\langle\downarrow\left|+e^{-i \pi / 4}\right| \downarrow\right\rangle\langle\uparrow| .
\end{aligned}
$$

(It is easily checked that the choices in (7)-(10) correspond to the choices Żukowski ( [15], p. 2) makes for the parameters he labels as $\beta, \phi, \beta^{\prime}, \phi^{\prime}$.) Żukowski shows that the following Bell-CHSH-type inequality

$$
\left|\begin{array}{c}
\left\langle A_{1} \otimes \sigma_{1}\right\rangle_{\left|\phi_{1}\right\rangle\left\langle\phi_{1}\right| \otimes D}+\left\langle A_{1} \otimes \sigma_{2}\right\rangle_{\left|\phi_{1}\right\rangle\left\langle\phi_{1}\right| \otimes D} \\
+\left\langle A_{2} \otimes \sigma_{1}\right\rangle_{\left|\phi_{2}\right\rangle\left\langle\phi_{2}\right| \otimes D}-\left\langle A_{2} \otimes \sigma_{2}\right\rangle_{\left|\phi_{2}\right\rangle\left\langle\phi_{2}\right| \otimes D}
\end{array}\right| \leq 2,
$$

which we henceforth for convenience call a "Bell teleportation inequality", is violated by a factor of $\sqrt{2}$. Żukowski appears to interpret this violation as signifying that the quantum component of a perfect teleportation process involving the maximally entangled state $D=\left|\Phi_{+}\right\rangle\left\langle\Phi_{+}\right|$must be nonlocal.

This interpretation is not without plausibility. The idea is that to assess the nonlocality of $D$ qua the quantum component of Alice's teleportation process, we should continue to have Alice measure the Bell operator but drop the classical channel, her phone call, from consideration. Although this frustrates any attempt to complete the process by Bob performing an appropriate unitary transformation on his qubit, it does not preclude asking whether what remains of the process can be modelled by a local hidden variables theory for any conceivable unknown state. One can imagine, then, that after preparing the quantum channel state, Clare randomly feeds to Alice one of two unknown states $\left|\phi_{j}\right\rangle$, and makes a covert agreement with Bob that he should simply ignore all of Alice's phone calls and randomly measure one of two spin components $\sigma_{j}$ on his qubit. After many measurement trials, Clare and Bob then reveal their charade to Alice, who grudgingly agrees to combine the information from her Bell operator outcomes with their information to see whether the Bell teleportation inequality (11) is violated. When they find that it is violated, the standard argument for Bell's theorem (cf. [15], Eqns. (16),(17)) shows that no hidden variables model according to which: (i) Alice's and Bob's measurement outcomes are statistically independent at the level of the hidden variables; (ii) Alice's outcomes are independent of the spin component Bob measures; and (iii) Bob's outcomes are independent of the unknown state Alice attempts to teleport, can possibly account for the observed correlations.

Right after pointing out that inequality (11) is violated in the state $D=\left|\Phi_{+}\right\rangle\left\langle\Phi_{+}\right|$, Żukowski remarks:

It is an interesting fact, that needs further investigation, that the Bell inequality presented here is violated by the same factor $\sqrt{2}$ as the CHSH inequality for the usual Bell theorem involving a pair of particles in a maximally entangled state. This may imply that the quantum component of the teleportation process cannot be described in a local and realistic way, as long as the initial state of $B$ [2] and $C[3]$ admits no such model ( [15], p. 3).

Żukowski's conjecture appears to be that if the channel state violates any local hidden variables theory inequality, perhaps even collective measurement ones, then the correlations involved in the quantum component of teleportation will also violate a local hidden variables theory inequality. If this is indeed the conjecture, it would be difficult to decide, since we would need to take into account all possible local hidden variables theories inequalities for all possible measurement protocols on the channel particles alone and determine whether a violation of any such inequality suffices to prevent the recovery, within a local hidden variables model, of the correlations of the channel state that are exploited in the teleportation process. Consequently, we narrow our focus to the standard Bell-CHSH inequality for the channel state and a generalized form of (11)'s Bell teleportation inequality for qubits 1-3 where the observables $A_{1}$ and $A_{2}$ are allowed to be any bivalent functions of Alice's Bell operator. 


\section{VIOLATION OF A BELL TELEPORTATION INEQUALITY IMPLIES VIOLATION OF A BELL-CHSH INEQUALITY BY THE CHANNEL STATE}

We first generalize Żukowski's scheme to include a wider class of observables compatible with Alice's Bell operator, by letting $A_{j}(j=1,2)$ be any pair of selfadjoint unitary 'spin' operators that are each bivalent functions of her Bell operator. Thus,

$$
\begin{aligned}
A_{j}= & a_{j}\left|\Psi_{+}\right\rangle\left\langle\Psi_{+}\left|+b_{j}\right| \Psi_{-}\right\rangle\left\langle\Psi_{-}\right| \\
& +c_{j}\left|\Phi_{+}\right\rangle\left\langle\Phi_{+}\left|+d_{j}\right| \Phi_{-}\right\rangle\left\langle\Phi_{-}\right|, j=1,2,
\end{aligned}
$$

where $a_{j}, b_{j}, c_{j}, d_{j}= \pm 1$. Now fix, once and for all, the channel state density operator $D$. By analogy with $\beta(D)$, define the number $\tau(D)$ to be the maximum of the Bell teleportation expression

$$
\left|\begin{array}{c}
\left\langle A_{1} \otimes \sigma_{1}\right\rangle_{\left|\phi_{1}\right\rangle\left\langle\phi_{1}\right| \otimes D}+\left\langle A_{1} \otimes \sigma_{2}\right\rangle_{\left|\phi_{1}\right\rangle\left\langle\phi_{1}\right| \otimes D} \\
+\left\langle A_{2} \otimes \sigma_{1}\right\rangle_{\left|\phi_{2}\right\rangle\left\langle\phi_{2}\right| \otimes D}-\left\langle A_{2} \otimes \sigma_{2}\right\rangle_{\left|\phi_{2}\right\rangle\left\langle\phi_{2}\right| \otimes D}
\end{array}\right|
$$

over all choices of the unknown state vectors $\left|\phi_{j}\right\rangle$ and all choices of the bivalent Bell operator functions $A_{j}$, i.e., all \pm 1 choices for the numbers $a_{j}, b_{j}, c_{j}, d_{j}$.

Notice that, since there is a unitary operator $U$ on the state space of qubit 1 mapping $\left|\phi_{2}\right\rangle$ to $\left|\phi_{1}\right\rangle$, (13) equals

$$
\left|\left\langle A_{1} \otimes\left(\sigma_{1}+\sigma_{2}\right)+\left(U A_{2} U^{-1}\right) \otimes\left(\sigma_{1}-\sigma_{2}\right)\right\rangle_{\left|\phi_{1}\right\rangle\left\langle\phi_{1}\right| \otimes D}\right|,
$$

which is just the absolute value of the expectation value of a Bell-CHSH operator on the bipartite system $(1+$ $2)+3$. Thus, necessarily, $\tau(D) \in[2,2 \sqrt{2}]$. Note also that if the unknown states $\left|\phi_{1}\right\rangle$ and $\left|\phi_{2}\right\rangle$ are compatible (i.e., either the same up to an irrelevant phase or orthogonal), then $U$ can always be chosen so that $U A_{2} U^{-1}$ is again a bivalent function of the Bell operator. This is most easily seen when $\left|\phi_{1}\right\rangle=|\uparrow\rangle$ and $\left|\phi_{2}\right\rangle=|\downarrow\rangle$, in which case the unitary that simply permutes these two $z$ eigenstates maps the four Bell operator eigenspaces into each other. (The general case can be reduced to this one by first applying a unitary transformation to the unknown states to bring them in line with the $z$-basis.) Thus, when the two alternative unknown states are compatible, $\left[A_{1}, U A_{2} U^{-1}\right]=0$. In that case, it follows that expression (14), and thus (13), cannot exceed 2, and the corresponding Bell teleportation inequality cannot be violated. This is to be expected, since it is known [16 that perfect teleportation of any collection of mutually compatible unknown states can be achieved with a separable channel state (all of whose correlations, including those involved in the teleportation, can be modelled by a local hidden variables theory).

It is not difficult to show that $\tau(D)=\beta(D)=2 \sqrt{2}$ if and only if $D$ is a maximally entangled pure state (by using the fact that any maximally entangled state is related to Żukowski's choice $D=\left|\Phi_{+}\right\rangle\left\langle\Phi_{+}\right|$by a unitary operator on Bob's qubit). We now concentrate on proving, quite generally, that $\beta(D) \geq \tau(D)$.

If $\tau(D)=2$ the claim is trivial, so we suppose $\tau(D)>$ 2 . Thus, there are unknown states $\left|\phi_{j}\right\rangle$ of qubit 1 , bivalent Bell operator functions $A_{j}$, and spin components $\sigma_{j}$ of qubit 3 such that

$$
\begin{aligned}
& \begin{array}{c}
\left\langle A_{1} \otimes \sigma_{1}\right\rangle_{\left|\phi_{1}\right\rangle\left\langle\phi_{1}\right| \otimes D}+\left\langle A_{1} \otimes \sigma_{2}\right\rangle_{\left|\phi_{1}\right\rangle\left\langle\phi_{1}\right| \otimes D} \\
+\left\langle A_{2} \otimes \sigma_{1}\right\rangle_{\left|\phi_{2}\right\rangle\left\langle\phi_{2}\right| \otimes D}-\left\langle A_{2} \otimes \sigma_{2}\right\rangle_{\left|\phi_{2}\right\rangle\left\langle\phi_{2}\right| \otimes D} \mid
\end{array} \\
& =\tau(D)>2 \text {. }
\end{aligned}
$$

Consider the pair of self-adjoint operators $X_{j}$ on qubit 2 's state space defined via the following matrix elements in the $\sigma_{z}$ eigenbasis:

$$
\left(\begin{array}{cc}
\left\langle\phi_{j}\left|\left\langle\uparrow\left|A_{j}\right| \phi_{j}\right\rangle\right| \uparrow\right\rangle & \left\langle\phi_{j}\left|\left\langle\uparrow\left|A_{j}\right| \phi_{j}\right\rangle\right| \downarrow\right\rangle \\
\left\langle\phi_{j}\left|\left\langle\downarrow\left|A_{j}\right| \phi_{j}\right\rangle\right| \uparrow\right\rangle & \left\langle\phi_{j}\left|\left\langle\uparrow\left|A_{j}\right| \phi_{j}\right\rangle\right| \uparrow\right\rangle
\end{array}\right), j=1,2 .
$$

Since each $A_{j}$ is self-adjoint, the matrices in (16) are selfadjoint; thus each $X_{j}$ is self-adjoint as well. By linearity, (16) implies:

$$
\left\langle v\left|X_{j}\right| w\right\rangle=\left\langle\phi_{j}\left|\left\langle v\left|A_{j}\right| \phi_{j}\right\rangle\right| w\right\rangle, j=1,2,
$$

for all qubit 2 vectors $|v\rangle,|w\rangle$. In particular, when we set both $|v\rangle$ and $|w\rangle$ equal to any unit eigenvector of $X_{j}$ with corresponding eigenvalue $\mu_{j}$, it follows that $\left|\mu_{j}\right| \leq$ $\left\|A_{j}\right\|=1$. Thus each $X_{j}$ is a self-adjoint contraction, i.e., satisfies $\left\|X_{j}\right\| \leq 1$.

Next, multiplying both sides of (17) by $\left\langle x\left|\sigma_{l}\right| y\right\rangle$, with $|x\rangle,|y\rangle$ arbitrary qubit 3 vectors, we obtain

$$
\begin{gathered}
\left\langle v\left|\left\langle x\left|X_{j} \otimes \sigma_{l}\right| w\right\rangle\right| y\right\rangle \\
=\left\langle\phi_{j}\left|\left\langle v\left|\left\langle x\left|A_{j} \otimes \sigma_{l}\right| \phi_{j}\right\rangle\right| w\right\rangle\right| y\right\rangle, j, l=1,2 .
\end{gathered}
$$

Now, since $D$ is Hermitian, $D=\sum_{k=1}^{4} \lambda_{k}\left|e_{k}\right\rangle\left\langle e_{k}\right|$, where the $\left\{e_{k}\right\}$ form an orthonormal basis for the $2+3$ space. Since product vectors span this space, (18) implies by linearity

$$
\begin{array}{r}
\left\langle e_{k}\left|X_{j} \otimes \sigma_{l}\right| e_{k}\right\rangle=\left\langle\phi_{j}\left|\left\langle e_{k}\left|A_{j} \otimes \sigma_{l}\right| \phi_{j}\right\rangle\right| e_{k}\right\rangle \\
k=1, \ldots, 4 ; j, l=1,2 .
\end{array}
$$

Multiplying both sides of (19) by $\lambda_{k}$ and summing over $k$ results in:

$$
\left\langle X_{j} \otimes \sigma_{l}\right\rangle_{D}=\left\langle A_{j} \otimes \sigma_{l}\right\rangle_{\left|\phi_{j}\right\rangle\left\langle\phi_{j}\right| \otimes D} j, l=1,2 .
$$

Combining this with (15) yields

$$
\left|\begin{array}{c}
\left\langle X_{1} \otimes \sigma_{1}\right\rangle_{D}+\left\langle X_{1} \otimes \sigma_{2}\right\rangle_{D} \\
+\left\langle X_{2} \otimes \sigma_{1}\right\rangle_{D}-\left\langle X_{2} \otimes \sigma_{2}\right\rangle_{D}
\end{array}\right|=\tau(D)>2
$$

Finally note that, although the $X_{j}$ are in general only self-adjoint contractions, convexity arguments [17] show 
that if a bipartite state (here, our channel state $D$ ) violates a Bell-CHSH-type inequality with respect to selfadjoint contractions (as in (21)), then that state also violates, by at least the same amount, a standard Bell$\mathrm{CHSH}$ inequality with respect to observables all of which are self-adjoint unitary spin components. This completes the proof that $\beta(D) \geq \tau(D)$.

An immediate corollary of this result is that there are channel states that permit nonclassical teleportation yet violate no Bell teleportation inequality. For example, consider the 'Werner state' 18

$$
D_{W}=\left(1-\frac{1}{\sqrt{2}}\right) \frac{1}{4} I \otimes I+\frac{1}{\sqrt{2}}\left|\Psi_{-}\right\rangle\left\langle\Psi_{-}\right| .
$$

Since spin operators, and hence Bell-CHSH operators, are traceless,

$$
\begin{aligned}
\beta\left(D_{W}\right) & =\frac{1}{\sqrt{2}} \beta\left(\left|\Psi_{-}\right\rangle\left\langle\Psi_{-}\right|\right) \\
& =\frac{1}{\sqrt{2}} 2 \sqrt{2}=2 \geq \tau\left(D_{W}\right)
\end{aligned}
$$

which forces $\tau\left(D_{W}\right)=2$. On the other hand, the teleportation fidelity of $D_{W}$ relative to the standard choice of Bob's unitary operators (which, in fact, gives the maximum achievable fidelity) is

$$
\begin{aligned}
\mathcal{F}_{s t}\left(D_{W}\right) & =\left(1-\frac{1}{\sqrt{2}}\right) \times \frac{1}{2}+\frac{1}{\sqrt{2}} \times 1 \\
& =\frac{2}{3}\left(1+\frac{3 \sqrt{2}-2}{8}\right)>\frac{2}{3} .
\end{aligned}
$$

\section{A CHANNEL STATE BELL-CHSH VIOLATION DOES NOT IMPLY A VIOLATION OF A BELL TELEPORTATION INEQUALITY.}

We now exhibit a family of channel states $D$ for which the converse inequality $\tau(D) \geq \beta(D)$ fails; indeed, for which $\beta(D)>2$ while $\tau(D)=2$. It follows that if one were to interpret Żukowski's conjecture (discussed in Section [II) to be that a channel state Bell-CHSH violation implies a violation of his original inequality, then this particular conjecture is false.

By the reasoning in the previous section which led to Eqn. (21), we know that the maximum Bell teleportation violation given a channel state $D$ is attained by an expression of the form $\left|\langle Z\rangle_{D}\right|$ where

$$
Z=X_{1} \otimes \sigma_{1}+X_{1} \otimes \sigma_{2}+X_{2} \otimes \sigma_{1}-X_{2} \otimes \sigma_{2},
$$

and the self-adjoint contractions $X_{j}(j=1,2)$ have matrix elements given by (16). We may calculate these matrix elements explicitly by parameterizing the two alternative teleported states as

$$
\left|\phi_{j}\right\rangle=\sin \theta_{j}|\uparrow\rangle+\cos \theta_{j} e^{i \vartheta_{j}}|\downarrow\rangle, \theta_{j}, \vartheta_{j} \in[0,2 \pi) .
$$

An elementary but tedious calculation then shows

$$
\begin{aligned}
X_{j}= & \frac{1}{4}\left(a_{j}+b_{j}+c_{j}+d_{j}\right) I \\
& +\frac{1}{4}\left(a_{j}-b_{j}+c_{j}-d_{j}\right) \sin 2 \theta_{j} \cos \vartheta_{j} \sigma_{x} \\
& +\frac{1}{4}\left(a_{j}+d_{j}-b_{j}-c_{j}\right) \sin 2 \theta_{j} \sin \vartheta_{j} \sigma_{y} \\
& +\frac{1}{4}\left(a_{j}+b_{j}-c_{j}-d_{j}\right) \cos 2 \theta_{j} \sigma_{z}
\end{aligned}
$$

where the numbers $a_{j}, b_{j}, c_{j}, d_{j}= \pm 1$ correspond to the different choices that can be made for the bivalent functions $A_{j}$ of Alice's Bell operator. The value of $\tau(D)$ is, therefore, given by

$$
\tau(D)=\max _{\theta_{j}, \vartheta_{j} \in[0,2 \pi) ; a_{j}, b_{j}, c_{j}, d_{j}= \pm 1 ; \sigma_{j}}\left|\langle Z\rangle_{D}\right| .
$$

Consider, now, the two parameter family of channel density operators given by

$$
D_{\lambda, \alpha}=(1-\lambda) \frac{1}{4} I \otimes I+\lambda\left|\psi_{\alpha}\right\rangle\left\langle\psi_{\alpha}\right|, \quad \lambda, \alpha \in[0,1],
$$

where

$$
\begin{gathered}
\left|\psi_{\alpha}\right\rangle=\alpha|+\rangle|+\rangle+\sqrt{1-\alpha^{2}}|-\rangle|-\rangle, \\
| \pm\rangle=\frac{1 \pm \sqrt{3}}{\sqrt{2(3 \pm \sqrt{3})}}|\uparrow\rangle+\frac{1+i}{\sqrt{2(3 \pm \sqrt{3})}}|\downarrow\rangle .
\end{gathered}
$$

For later reference, note that

$$
\begin{aligned}
\left\langle\psi_{\alpha}\left|I \otimes \sigma_{n}\right| \psi_{\alpha}\right\rangle & =\frac{1}{\sqrt{3}}\left(2 \alpha^{2}-1\right), n=x, y, z, \\
\left\langle\psi_{\alpha}\left|\sigma_{m} \otimes \sigma_{n}\right| \psi_{\alpha}\right\rangle & =\frac{1}{3}\left(1+4 \alpha \sqrt{1-\alpha^{2}}\right) \\
(m, n) & =(x, y),(y, x),(z, z) \\
\left\langle\psi_{\alpha}\left|\sigma_{m} \otimes \sigma_{m}\right| \psi_{\alpha}\right\rangle & =\left\langle\psi_{\alpha}\left|\sigma_{m} \otimes \sigma_{z}\right| \psi_{\alpha}\right\rangle \\
& =\left\langle\psi_{\alpha}\left|\sigma_{z} \otimes \sigma_{m}\right| \psi_{\alpha}\right\rangle \\
& =\frac{1}{3}\left(1-2 \alpha \sqrt{1-\alpha^{2}}\right), m=x, y
\end{aligned}
$$

Since spin operators are traceless, the corresponding expectation values in the state $D_{\lambda, \alpha}$ are obtained by multiplying each of the above values by $\lambda$.

Again, because spin operators are traceless, the first maximally mixed component of $D_{\lambda, \alpha}$ does not contribute to any Bell-CHSH operator average; hence $\beta\left(D_{\lambda, \alpha}\right)>2$ just in case $\lambda \beta\left(\left|\psi_{\alpha}\right\rangle\left\langle\psi_{\alpha}\right|\right)>2$. It can be shown that [1]

$$
\beta\left(\left|\psi_{\alpha}\right\rangle\left\langle\psi_{\alpha}\right|\right)=2 \sqrt{1+4 \alpha^{2}\left(1-\alpha^{2}\right)},
$$


thus the condition for $\beta\left(D_{\lambda, \alpha}\right)>2$ is simply

$$
\lambda \sqrt{1+4 \alpha^{2}\left(1-\alpha^{2}\right)}>1, \lambda, \alpha \in[0,1] .
$$

We now determine two additional conditions on $\lambda$ and $\alpha$, consistent with (36), which together are sufficient for $\tau\left(D_{\lambda, \alpha}\right)=2$.

The set of 16 possible value assignments to the numbers $a_{j}, b_{j}, c_{j}, d_{j}$ can be divided into three relevant classes. Class I: All assignments for which both $A_{1}$ and $A_{2}$ involve two value assignments of both signs (for example, the two value assignments $a_{j}=b_{j}=-c_{j}=-d_{j}=$ -1 , where $j=1,2)$. Class II: All assignments for which exactly one of $A_{1}$ and $A_{2}$ involve two value assignments of both signs and the other involves three values assignments of one sign (for example, $a_{1}=b_{1}=-c_{1}=-d_{1}=$ -1 and $a_{2}=b_{2}=c_{2}=-d_{2}=-1$ ). Class III: All assignments for which both $A_{1}$ and $A_{2}$ involve exactly three values assignments of one sign (for example, the value assignments $a_{j}=b_{j}=c_{j}=-d_{j}=-1$ ). Note that whenever $a_{j}=b_{j}=c_{j}=d_{j}$ for at least one value of $j$-i.e., whenever either $A_{1}$ or $A_{2}$ is multiple of the identity - no violation is possible, thus such assignments can be ignored.

Class I: Following [1], we introduce the new spin operators

$$
\hat{\sigma}_{1}=\frac{\sigma_{1}+\sigma_{2}}{2 \cos \xi}, \hat{\sigma}_{2}=\frac{\sigma_{1}-\sigma_{2}}{2 \sin \xi},
$$

so that (25) becomes

$$
Z=2\left(X_{1} \otimes \hat{\sigma}_{1}\right) \cos \xi+2\left(X_{2} \otimes \hat{\sigma}_{2}\right) \sin \xi .
$$

Using the fact that, whenever $M, N \in \mathbb{R}, M \cos \xi+$ $N \sin \xi \leq \sqrt{M^{2}+N^{2}}$, we have

$$
\left|\langle Z\rangle_{D_{\lambda, \alpha}}\right| \leq 2 \sqrt{\left\langle X_{1} \otimes \hat{\sigma}_{1}\right\rangle_{D_{\lambda, \alpha}}^{2}+\left\langle X_{2} \otimes \hat{\sigma}_{2}\right\rangle_{D_{\lambda, \alpha}}^{2}} .
$$

Using similar reasoning,

$$
\left\langle X_{j} \otimes \hat{\sigma}_{j}\right\rangle_{D_{\lambda, \alpha}} \leq \sqrt{\sum_{n=x, y, z}\left\langle X_{j} \otimes \sigma_{n}\right\rangle_{D_{\lambda, \alpha}}^{2}}, j=1,2,
$$

therefore

$$
\left|\langle Z\rangle_{D_{\lambda, \alpha}}\right| \leq 2 \sqrt{\sum_{n=x, y, z ; j=1,2}\left\langle X_{j} \otimes \sigma_{n}\right\rangle_{D_{\lambda, \alpha}}^{2}} .
$$

Assuming a Class I value assignment to the numbers $a_{j}, b_{j}, c_{j}, d_{j}$, each self-adjoint contraction in 27) has the form $X_{j}=r_{j} \sigma_{m_{j}}$ where $r_{j} \in[-1,1]$ and $m_{j}=x, y, z$. Since $\left|\langle Z\rangle_{D_{\lambda, \alpha}}\right|$ cannot exceed the classical bound 2 when $\left[X_{1}, X_{2}\right]=0$, we need only consider the three cases where $m_{1} \neq m_{2}$, i.e.,

$$
\begin{aligned}
& X_{1}=r_{1} \sigma_{x}, X_{2}=r_{2} \sigma_{y}, \\
& X_{1}=r_{1} \sigma_{y}, X_{2}=r_{2} \sigma_{z}, \\
& X_{1}=r_{1} \sigma_{x}, X_{2}=r_{2} \sigma_{z},
\end{aligned}
$$

and the other three cases obtained by interchanging 1 and 2. For the first case, substitution into inequality (41) and using the expectation values given in (33) and (34), we find

$$
\begin{aligned}
\left|\langle Z\rangle_{D_{\lambda, \alpha}}\right| & \leq \frac{2 \lambda}{3} \sqrt{\left(r_{1}^{2}+r_{2}^{2}\right)\left(3+24 \alpha^{2}\left(1-\alpha^{2}\right)\right)} \\
& \leq \frac{2 \sqrt{2} \lambda}{\sqrt{3}} \sqrt{1+8 \alpha^{2}\left(1-\alpha^{2}\right)}
\end{aligned}
$$

The other two cases in (42) yield the same inequality (45), as do the cases obtained by interchanging 1 and 2 (which leaves the right-hand side of inequality (41) unchanged). Thus, a sufficient condition for no Class I value assignment to yield a violation of a Bell teleportation inequality is

$$
\lambda \sqrt{2 / 3\left(1+8 \alpha^{2}\left(1-\alpha^{2}\right)\right)} \leq 1 .
$$

Class II: In this case, we can see from (27) that exactly one of $X_{1}$ and $X_{2}$ takes the form $r \sigma_{m}$, where $m=x, y, z$ and $r \in[-1,1]$, while the other is of the form $\pm I / 2+\sigma / 2$ where $\sigma$ is a spin- $1 / 2$ operator. Thus,

$$
\begin{aligned}
Z & =r \sigma_{m} \otimes\left(\sigma_{2}+\bar{\sigma}_{2}\right)+1 / 2(\sigma \pm I) \otimes\left(\sigma_{1}-\bar{\sigma}_{2}\right) \\
& = \pm I \otimes\left(\sigma_{1}-\bar{\sigma}_{2}\right) / 2+r \sigma_{m} \otimes\left(\sigma_{1}+\bar{\sigma}_{2}\right) / 2+B^{\prime} / 2,
\end{aligned}
$$

where $\bar{\sigma}_{2}= \pm \sigma_{2}$ and $B^{\prime}$ is a Bell-CHSH-type operator constructed out of self-adjoint contractions that are not necessarily unitary (unless $r= \pm 1$ ). Transforming the spin operators $\left(\sigma_{1}, \bar{\sigma}_{2}\right) \mapsto\left(\hat{\sigma}_{1}, \hat{\sigma}_{2}\right)$ via Eqns. (37), and substituting into Eqn. (47) yields

$$
Z= \pm\left(I \otimes \hat{\sigma}_{2}\right) \sin \xi+r\left(\sigma_{m} \otimes \hat{\sigma}_{1}\right) \cos \xi+B^{\prime} / 2 .
$$

Therefore,

$$
\begin{gathered}
\left|\langle Z\rangle_{D_{\lambda, \alpha}}\right| \\
\leq \quad \mid \pm\left\langle I \otimes \hat{\sigma}_{2}\right\rangle_{D_{\lambda, \alpha}} \sin \xi \\
\leq \quad \sqrt{\left.+\sigma_{m} \otimes \hat{\sigma}_{1}\right\rangle_{D_{\lambda, \alpha}} \cos \xi|+1 / 2|\left\langle B^{\prime}\right\rangle_{D_{\lambda, \alpha}} \mid} \\
\leq \sqrt{\left\langle I \otimes \hat{\sigma}_{2}\right\rangle_{D_{\lambda, \alpha}}^{2}+r^{2}\left\langle\sigma_{m} \otimes \hat{\sigma}_{1}\right\rangle_{D_{\lambda, \alpha}}^{2}} \\
+1 / 2\left|\left\langle B^{\prime}\right\rangle_{D_{\lambda, \alpha}}\right| .
\end{gathered}
$$

¿From (35) it follows that

$$
\left|\left\langle B^{\prime}\right\rangle_{D_{\lambda, \alpha}}\right| \leq 2 \lambda \sqrt{1+4 \alpha^{2}\left(1-\alpha^{2}\right)}
$$

and, clearly,

$$
r^{2}\left\langle\sigma_{m} \otimes \hat{\sigma}_{1}\right\rangle_{D_{\lambda, \alpha}}^{2}=\lambda^{2} r^{2}\left\langle\sigma_{m} \otimes \hat{\sigma}_{1}\right\rangle_{\left|\psi_{\alpha}\right\rangle\left\langle\psi_{\alpha}\right|} \leq \lambda^{2} .
$$

Moreover, 


$$
\begin{gathered}
\left\langle I \otimes \hat{\sigma}_{2}\right\rangle_{D_{\lambda, \alpha}}^{2} \\
\leq\left\langle I \otimes \sigma_{x}\right\rangle_{D_{\lambda, \alpha}}^{2}+\left\langle I \otimes \sigma_{y}\right\rangle_{D_{\lambda, \alpha}}^{2}+\left\langle I \otimes \sigma_{z}\right\rangle_{D_{\lambda, \alpha}}^{2} \\
=\quad \lambda^{2}\left(2 \alpha^{2}-1\right)^{2},
\end{gathered}
$$

using Eqn. (32) for the last equality. Upon substitution of these last three inequalities back into inequality (49), we obtain

$$
\begin{aligned}
\left|\langle Z\rangle_{D_{\lambda, \alpha}}\right| \leq & \lambda\left(\sqrt{2\left(1-2 \alpha^{2}\left(1-\alpha^{2}\right)\right)}\right. \\
& \left.+\sqrt{1+4 \alpha^{2}\left(1-\alpha^{2}\right)}\right)
\end{aligned}
$$

Thus, a sufficient condition for no Class II value assignment to yield a violation of a Bell teleportation inequality is

$$
\lambda\left(\sqrt{2\left(1-2 \alpha^{2}\left(1-\alpha^{2}\right)\right)}+\sqrt{1+4 \alpha^{2}\left(1-\alpha^{2}\right)}\right) \leq 2 .
$$

Class III: In this case, $Z$ takes the form $I \otimes \sigma+B / 2$ where $\sigma$ is a spin-operator and $B$ a standard Bell-CHSH operator. Note that this is just a special case of the Class II expression for $Z$ in Eqn. (48), taking the \pm sign to be $+, \hat{\sigma}_{2}=\sigma, \xi=\pi / 2$, and $B^{\prime}=B$. Thus inequality (54) is also sufficient for no Class III value assignment to yield a violation of a Bell teleportation inequality.

Combining our results for each class, we see that $\beta\left(D_{\lambda, \alpha}\right)>2$ while $\tau\left(D_{\lambda, \alpha}\right)=2$ if and only if $\lambda$ and $\alpha$ satisfy conditions (36), (46) and (54). The family of channel density operators satisfying these conditions is indeed nonempty. For example, the conditions are satisfied by taking $\lambda=\sqrt{3 / 5} \approx .77$ and $\alpha=\sqrt{3} / 2 \approx .87$, and in that case we have $\beta\left(D_{\sqrt{3 / 5}, \sqrt{3} / 2}\right)=2 \sqrt{21 / 20} \approx 2.05$. Note, also, that choosing $\lambda=1$ does not satisfy the conditions, nor does choosing $\alpha^{2}\left(1-\alpha^{2}\right)=1 / 4$. Thus no channel density operator in the family is pure, and the pure entangled component $\left|\psi_{\alpha}\right\rangle\left\langle\psi_{\alpha}\right|$ of $D_{\lambda, \alpha}$ is never maximally entangled. Finally, since $\beta(D)>2$ implies $\mathcal{F}_{\max }(D)>2 / 3$ [7], each density operator in the family supplies another example of a state that permits nonclassical teleportation without violating any Bell teleportation inequality.

\section{FIDELITY VALUES THAT IMPLY A VIOLATION OF A BELL TELEPORTATION INEQUALITY.}

In light of our previous results, it is natural to ask what values of the fidelity are sufficient for the quantum correlations in the standard teleportation protocol not to admit a local hidden variables explanation. We shall show in this section that $\mathcal{F}_{s t}(D)>2 / 3(1+1 / 2 \sqrt{2}) \approx .90$ implies $\tau(D)>2$.

It is known [19] that for any Bell-CHSH operator $B$,

$$
\langle B\rangle_{D}=\mathbf{a} \cdot \mathbf{T}(\mathbf{D})\left(\mathbf{b}+\mathbf{b}^{\prime}\right)+\mathbf{a}^{\prime} \cdot \mathbf{T}(\mathbf{D})\left(\mathbf{b}-\mathbf{b}^{\prime}\right)
$$

where $\mathbf{a}, \mathbf{a}^{\prime}, \mathbf{b}$ and $\mathbf{b}^{\prime}$ are unit vectors in $\mathbb{R}^{3}$ defining the four spin operators that occur in $B$, and the $3 \times 3$ matrix $T(D)$ has components

$$
T_{m n}(D)=\operatorname{Tr}\left[D\left(\sigma_{m} \otimes \sigma_{n}\right)\right], m, n=x, y, z,
$$

encoding the inter-particle correlations in state $D$. Setting $\mathbf{a}=\mathbf{x}, \mathbf{a}^{\prime}=\mathbf{y}, \mathbf{b}=(\mathbf{x}+\mathbf{y}) / \sqrt{\mathbf{2}}$, and $\mathbf{b}^{\prime}=(\mathbf{x}-\mathbf{y}) / \sqrt{\mathbf{2}}$, we obtain

$$
\begin{aligned}
\langle B\rangle_{D} & =\left\langle\sigma_{x} \otimes \sigma_{b}+\sigma_{x} \otimes \sigma_{b^{\prime}}+\sigma_{y} \otimes \sigma_{b}-\sigma_{y} \otimes \sigma_{b^{\prime}}\right\rangle_{D} \\
& =\sqrt{2}\left(T_{x x}(D)+T_{y y}(D)\right) .
\end{aligned}
$$

Now choose the operators $A_{j}$ in Eqn. (12) that correspond to $a_{1}=c_{1}=-b_{1}=-d_{1}=+1$ and $a_{2}=d_{2}=$ $-b_{2}=-c_{2}=+1$, and the unknown states $\left|\phi_{j}\right\rangle$ in (26) that correspond to $\theta_{1}=\pi / 4, \vartheta_{1}=0, \theta_{2}=\pi / 4$, and $\vartheta_{2}=\pi / 4$. Substituting these values into (27), we obtain $X_{1}=\sigma_{x}$ and $X_{2}=\sigma_{y}$. Hence the Bell-CHSH operator $B$ in (57) has the form of a $Z$ operator as given in (25), and $\left|\langle B\rangle_{D}\right|$ provides a lower bound for $\tau(D)$, i.e.,

$$
\tau(D) \geq \sqrt{2}\left|T_{x x}(D)+T_{y y}(D)\right| .
$$

(As check on the correctness of this bound, note from (34), that it falls below 2 when $D=D_{.77 .87}$, as it must.)

It can be shown quite generally that []

$$
\mathcal{F}_{s t}(D)=\frac{1}{8} \sum_{n=1}^{4}\left(1+\frac{1}{3} \operatorname{Tr}\left[T_{n}^{\dagger} T(D) O_{n}\right]\right)
$$

where

$$
\begin{array}{r}
T_{1}=\operatorname{diag}(-1,-1,-1), T_{2}=\operatorname{diag}(-1,1,1), \\
T_{3}=\operatorname{diag}(1,-1,1), T_{4}=\operatorname{diag}(1,1,-1),
\end{array}
$$

and the matrices $\left\{O_{n}\right\}$ are determined by the standard choice for Bob's unitary operations in (3) via the requirement that $U_{n}(\mathbf{b} \cdot \sigma) \mathbf{U}_{\mathbf{n}}^{-\mathbf{1}}=\left(\mathbf{O}_{\mathbf{n}}^{\dagger} \mathbf{b}\right) \cdot \sigma$ for all $\mathbf{b} \in \mathbb{R}^{\mathbf{3}}$. Calculating out these latter matrices explicitly yields

$$
\begin{gathered}
O_{1}=\operatorname{diag}(1,1,1), O_{2}=\operatorname{diag}(1,-1,-1), \\
O_{3}=\operatorname{diag}(-1,1,-1), O_{4}=\operatorname{diag}(-1,-1,1) .
\end{gathered}
$$

(For example, for $n=2, U_{2}=\sigma_{x}$, and, using the fact that $\sigma_{x}^{2}=I$ and orthogonal spin components anti-commute,

$$
\begin{aligned}
\sigma_{x}(\mathbf{b} \cdot \sigma) \sigma_{\mathbf{x}} & =\sigma_{x}\left(b_{x} \sigma_{x}+b_{y} \sigma_{y}+b_{z} \sigma_{z}\right) \sigma_{x} \\
& =b_{x} \sigma_{x}-b_{y} \sigma_{y}-b_{z} \sigma_{z} \\
& =\left(O_{2}^{\dagger} \mathbf{b}\right)_{\mathbf{x}} \sigma_{\mathbf{x}}+\left(\mathbf{O}_{2}^{\dagger} \mathbf{b}\right)_{\mathbf{y}} \sigma_{\mathbf{y}}+\left(\mathbf{O}_{\mathbf{2}}^{\dagger} \mathbf{b}\right)_{\mathbf{z}} \sigma_{\mathbf{z}}
\end{aligned}
$$

which implies $O_{2}=\operatorname{diag}(1,-1,-1)$.) Plugging all the matrices $\left\{T_{n}\right\}$ and $\left\{O_{n}\right\}$ back into (59) gives

$$
\mathcal{F}_{s t}(D)=1 / 2-1 / 6\left(T_{x x}(D)+T_{y y}(D)+T_{z z}(D)\right) .
$$

Finally, combining (58) with Eqn. (67), we have 


$$
\tau(D) \geq \sqrt{2}\left|T_{z z}(D)+6 \mathcal{F}_{s t}(D)-3\right| .
$$

Assuming $\mathcal{F}_{s t}(D)>2 / 3(1+1 / 2 \sqrt{2})=2 / 3+\sqrt{2} / 6$, and noting that $T_{z z}(D) \geq-1$, it follows that

$$
\begin{gathered}
\sqrt{2}\left(T_{z z}(D)+6 \mathcal{F}_{s t}(D)-3\right) \\
>\sqrt{2}(-1+4+\sqrt{2}-3)=2,
\end{gathered}
$$

and therefore $\tau(D)>2$, as claimed. Note that we have made no attempt to find the minimum value for $\mathcal{F}_{s t}(D)$ that implies $\tau(D)>2$. However, for the Werner state in (22), we know that $\mathcal{F}_{s t}\left(D_{W}\right)=2 / 3(1+(3 \sqrt{2}-2) / 8) \approx$ .85 , yet $\tau\left(D_{W}\right)=2$; so our bound of .90 cannot be decreased below 85 .

\section{DISCUSSION}

We have compared a new class of "Bell teleportation inequalities" - our generalization of Żukowski's inequality - to the well-known class of Bell-CHSH inequalities for the particle pair that makes up the quantum channel of a standard teleportation process. We found that while a Bell teleportation inequality cannot be violated unless the channel state already violates a Bell-CHSH inequality, the latter is no guarantee that the correlations of the channel state actually involved in the teleportation process will violate a Bell teleportation inequality. This suggests that it is generally easier for a local hidden variables theory to simulate teleportation than to simulate the results of a standard Bell correlation experiment on the channel particles alone. Moreover, if one were to interpret Żukowski's conjecture (discussed in Section III to be that a channel state Bell-CHSH violation implies a violation of his original inequality, then we have shown this particular conjecture to be false.

Our results also contribute to the larger debate (discussed in our introduction) over the role that nonlocality plays in teleportation. We showed that a Bell teleportation inequality is always violated when the fidelity of transmission in the standard teleportation protocol exceeds the classical limit of $2 / 3$ by a factor of $1+1 / 2 \sqrt{2}$. If one accepts that the violation of an inequality to which any local hidden variables theory is committed implies the presence of nonlocality, it follows that standard teleportation with a fidelity exceeding $2 / 3(1+1 / 2 \sqrt{2}) \approx .90$ cannot occur without the involvement of nonlocality. On the other hand, our results suggest the existence of a range $(.67, .90)$ of nonclassical values of the fidelity for which a local hidden variables theory of the teleportation process may be possible. Thus, the ability of an entangled channel state, such as $D_{W}$ or $D_{.77, .87}$, to permit nonclassical teleportation cannot by itself suffice for concluding that the channel state itself, or the teleportation it facilitates, is nonlocal; attention must also be paid to the magnitude of the fidelity achievable using the state.
Our tentative conclusion that local hidden variables models of quantum teleportation may exist for nonclassical fidelities up to $\approx 0.90$ is compatible with Gisin's [6] demonstration that the end results of quantum teleportation can be classically simulated up to a fidelity of $\approx 0.87$. However, this comparison should not be given too much weight because Gisin's simulation has little to do with what actually goes on in the standard quantum teleportation protocol. In his simulation, Alice does not measure any Bell operator on qubits $1+2$. Rather, it is assumed that she has full knowledge of the 'unknown' state's expansion coefficients, and her task is simply to classically communicate to Bob as much information as she can about these coefficients by using only 2 bits. While it is certainly of interest to study how well teleportation can be achieved classically using various protocols different from the standard quantum protocol (see also [20]), we have confined ourselves in this paper to the possibility of local classical explanations of the latter only. While we have reached a negative conclusion for fidelities exceeding $\approx .90$, our results strongly suggest a positive answer for fidelities in the range $(.67, .90)$.

\section{Acknowledgements}

DTP acknowledges the support of a Graduate School Research Travelling Award from the University of Queensland and thanks the Department of Philosophy at the University of Pittsburgh for its hospitality. We would also like to thank Peter Drummond, Gerard Milburn, and William Munro for helpful discussion.

[1] S. Popescu, D. Rohrlich, Phys. Lett. A 166 (1992) 293.

[2] C. H. Bennett, G. Brassard, C. Crépeau, R. Jozsa, A. Peres, and W. K. Wooters, Phys. Rev. Lett. 70 (1993) 1895.

[3] S. L. Braunstein, A. Mann, M. Revzen, Phys. Rev. Lett. 68 (1992) 3259.

[4] R. F. Werner, quant-ph/0003070.

[5] S. Popescu, Phys. Rev. Lett. 72 (1994) 797.

[6] N. Gisin, Phys. Lett. A 210 (1996) 157.

[7] R. Horodecki, M. Horodecki, and P. Horodecki, quant-ph/9606027.

[8] S. Massar and S. Popescu, Phys. Rev. Lett. 74 (1995) 1259.

[9] L. Vaidman, quant-ph/9810089.

[10] J. Bub, Stud. Hist. Phil. Mod. Phys. 31 (2000) 74.

[11] O. Maroney and B. J. Hiley, Found. Phys. 29 (1999) 1403.

[12] L. Hardy, quant-ph/9906123.

[13] D. Deutsch and P. Hayden, quant-ph/9906007.

[14] A. Duwell, "Explaining Information Transfer in Quantum Teleportation", Phil. Sci. (2001) forthcoming. 
[15] M. Żukowski, Phys. Rev. A 62 (2000) 032101.

[16] S. Ghosh, G. Kar, A. Roy, and U. Sen, quant-ph/0010012.

[17] R. Clifton, H. Halvorson, and A. Kent, Phys. Rev. A 61 (2000) 042101.
[18] R. F Werner, Phys. Rev. A, 40 (1989) 4277.

[19] R. Horodecki, P. Horodecki, and M. Horodecki, Phys. Lett. A 200 (1995) 340.

[20] N. Cerf, N. Gisin, and S. Massar, Phys. Rev. Lett., 84 (2000) 2521. 